\title{
ESIPT-based fluorescence probe for the ratiometric detection of superoxide
}

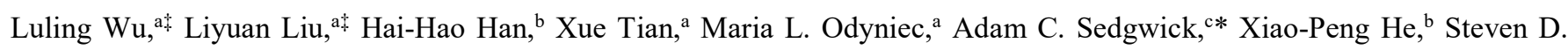
Bull, ${ }^{\text {a* }}$ and Tony D. James ${ }^{\mathrm{a} *}$

\begin{abstract}
a. Department of Chemistry, University of Bath, Bath, BA2 7AY,UK. Email: t.d.james@bath.ac.uk, s.d.bull@bath.ac.uk
b. Key Laboratory for Advanced Materials and Joint International Research Laboratory of Precision Chemistry and Molecular Engineering, Feringa Nobel Prize Scientist Joint Research Center, School of Chemistry and Molecular Engineering, East China University of Science and Technology, 130 Meilong Rd., Shanghai 200237, China.
\end{abstract}

c. Department of Chemistry, University of Texas at Austin, 105 E 24th street A5300, Austin, TX 78712-1224 (USA). Email: a.c.sedgwick@utexas.edu

A simple ESIPT-based fluorescence probe (HMBT-LW) was developed for the detection of superoxide $\left(\mathrm{O}_{2}{ }^{\circ}{ }^{-}\right)$. $\mathrm{HMBT}^{-\mathrm{LW}}$ was synthesised over two steps and was shown to rapidly detect low concentrations of $\mathrm{O}_{2}{ }^{-}$(limit of detection $=7.4 \mu \mathrm{M}$ ), fully reacting within two minutes. Furthermore, HMBT-LW demonstrated excellent selectivity and sensitivity towards $\mathrm{O}_{2}{ }^{\circ}$.

Reactive oxygen species (ROS) are the transient by-products generated from the electron transport chain. ${ }^{1}$ More specifically, the ROS, superoxide $\left(\mathrm{O}_{2}{ }^{-}\right)$is a anion radical generated from the single electron reduction of molecular oxygen $\left(\mathrm{O}_{2}\right)$, which means $\mathrm{O}_{2}{ }^{-}$ is the precursor to most $\mathrm{ROS}^{2} \mathrm{O}_{2}{ }^{-\cdots}$ is capable of reacting with nitric oxide (NO'), which generates the highly reactive nitrogen species peroxynitrite $\left(\mathrm{ONOO}^{-}\right)$, or with superoxide distmutase (SOD) to produce hydrogen peroxide $\left(\mathrm{H}_{2} \mathrm{O}_{2}\right)$. Hydrogen peroxide can then be transformed into the highly reactive hydroxyl radical $(\bullet \mathrm{OH})$ and hypochlorous acid (HOCl). These reactive oxygen species are associated with a number of pathological processes, including cardiomyopathy, autism, diabetes mellitus, cancer and neurodegenerative disorders (e.g., Alzheimer's disease and Parkinson's disease). ${ }^{3-6}$ Therefore, the development of a fluorescent probe for the real-time detection of $\mathrm{O}_{2}{ }^{-}$- would further aid the understanding of $\mathrm{O}_{2}{ }^{-}$- related diseases in living organisms.

Excited-state intramolecular proton transfer (ESIPT) is widely used in the design of fluorescent probes ${ }^{7}$ as ESIPT-based fluorescence probes display a number of favourable properties such as a large Stokes shift ( $200 \mathrm{~nm})$ and the ability to undergo ratiometric sensing. The ratiometric detection of a target analyte is ideal as it enables the determination of the concentration of the target analyte directly without need of calibration. ${ }^{7}$

Within our research group, we have developed several ESIPT-based fluorescence probes for the detection of biological reactive oxygen species as well as biological thiols. ${ }^{8-11}$ Previously, we have developed a thiocarbamate functionalised methoxyhydroxybenzothiazole (HMBT) fluorescent probe TCBT-OMe for the detection of $\mathrm{HOCl} / \mathrm{ClO}^{-}$(Scheme 1). The addition of $\mathrm{HOCl} /$ $\mathrm{ClO}^{-}$to TCBT-OMe resulted in the rapid hydrolysis $(<10 \mathrm{~s})$ of the thiocarbamate linker, leading to a ratiometric change in fluorescence intensity 10

Most fluorescent probes that are reported for the detection of $\mathrm{O}_{2}{ }^{--}$utilise its nucleophilicity to achieve excellent selectivity over other ROS. ${ }^{12-17}$ As a result of this, we believed the functionalisation of HMBT with the $\mathrm{O}_{2}{ }^{--}$reactive trifluoromethanesulfonate unit would result in a ratiometric fluorescent probe for the detection of $\mathrm{O}_{2}{ }^{\cdot-}$ (Scheme 1). ${ }^{18}$

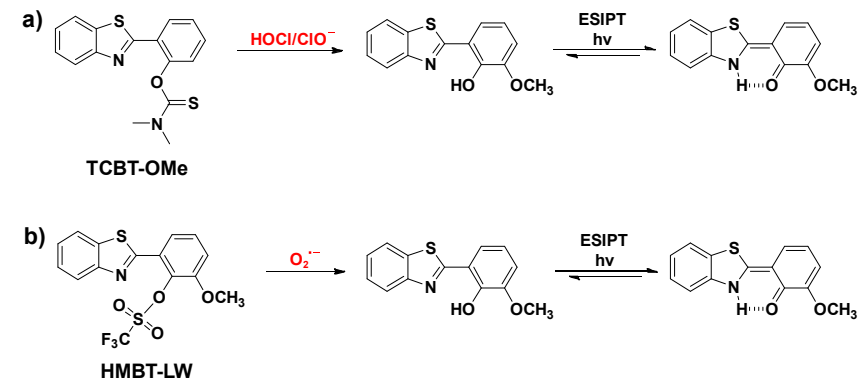

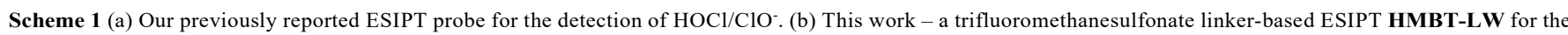
detection of $\mathrm{O}_{2} \cdot$

HMBT-LW was synthesized over two steps. The first step of the synthesis involved the addition of a $2: 1 \mathrm{aq} \mathrm{H}_{2} \mathrm{O}_{2} / \mathrm{aq} \mathrm{HCl}_{\mathrm{solution}}$ to 2-aminothiophenol and $o$-vanilin in $\mathrm{EtOH}$, which formed HMBT in good yield $(68 \%) .{ }^{19}$ With HMBT in hand, trifluoromethanesulfonic anhydride was then added dropwise into a solution of HMBT in DCM at $-78{ }^{\circ} \mathrm{C}$ under argon, $\mathrm{NEt}_{3}$ was subsequently added to the reaction. This reaction proceeded smoothly furnishing HMBT-LW in good yield (52 \%) (Scheme S2). 
The chemical structure of HMBT-LW was fully characterized by ${ }^{1} \mathrm{H}$ NMR, ${ }^{13} \mathrm{C}$ NMR and high-resolution mass spectrometry (HRMS).

Next, we evaluated the UV-Vis properties of HMBT-LW $(5 \mu \mathrm{M})$ with the addition of $\mathrm{O}_{2}-$ ( 39 equivalents). This addition led to an increase in UV absorption between 200 - $400 \mathrm{~nm}$ indicating a change chemical structure (Fig. S1). We then turned our attention towards the ability of HMBT-LW to detect $\mathrm{O}_{2}-$ using fluorescence. Remarkably, HMBT-LW was shown to have a rapid response towards $\mathrm{O}_{2}{ }^{--}$with a significant increase in fluorescence intensity being observed within 2 minutes (Fig. S2). Initially, a fluorescence emission intensity at $378 \mathrm{~nm}$ was only observed, since the ESIPT process is blocked by the trifluoromethanesulfonate group. However, in the presence of $\mathrm{O}_{2}{ }^{--}$, a notable increase in fluorescence emission intensity at $483 \mathrm{~nm}$ and a simultaneously decrease in fluorescence emission intensity at $378 \mathrm{~nm}$ was observed corresponding to the deprotection and release of the HMBT fluorophore enabling the ESIPT process to take place (Reaction mechanism confirmed by HRMS - see Fig. S3-4).
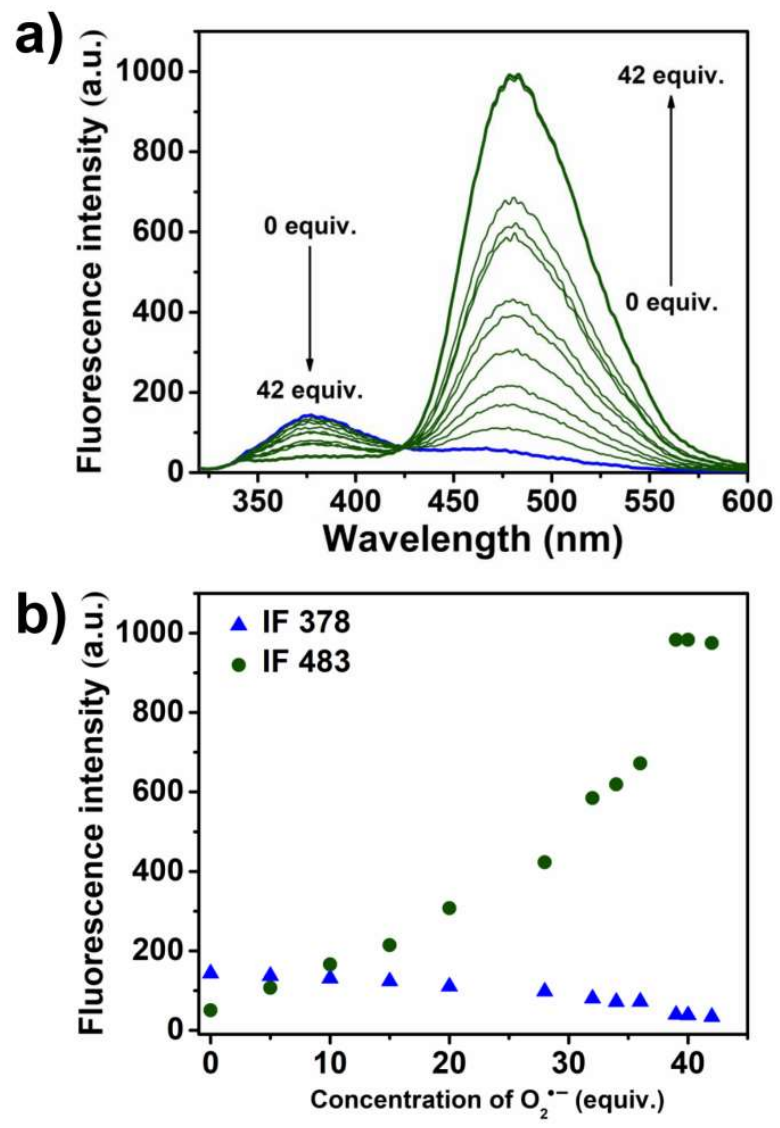

Fig. 1 (a) Changes in fluorescence emission intensity of HMBT-LW $(5 \mu \mathrm{M})$ with increasing additions of $\mathrm{O}_{2}{ }^{-}$(from 0 to 42 equiv.) in PBS buffer solution (10 mM, V/V, DMSO/PBS $=1 / 1, \mathrm{pH}=7.4$ ) after $3 \mathrm{~min}$. (b) Emission at 378 and $483 \mathrm{~nm}$ of HMBT-LW $(5 \mu \mathrm{M})$ with increasing addition of $\mathrm{O}_{2}{ }^{\circ}$ (from 0 to 42 equiv.) in PBS buffer solution $(10 \mathrm{mM}, \mathrm{V} / \mathrm{V}$, $\mathrm{DMSO} / \mathrm{PBS}=1 / 1, \mathrm{pH}=7.4$ ) after $3 \mathrm{~min} . \lambda_{\mathrm{ex}}=310 \mathrm{~nm}$. Slit widths: $\mathrm{ex}=8 \mathrm{~nm}$, em $=5 \mathrm{~nm}$.

HMBT-LW was then shown to have good stability over a range of different $\mathrm{pH} 4$ - 10, (Fig. S5) and was capable of detecting low concentrations of $\mathrm{O}_{2}{ }^{--}$with a Limit of Detection (LoD) of $7.4 \mu \mathrm{M}$ (Fig. S4). Furthermore, HMBT-LW demonstrated excellent selectivity towards $\mathrm{O}_{2}{ }^{-*}$ over other ROS and biologically relevant analytes. 


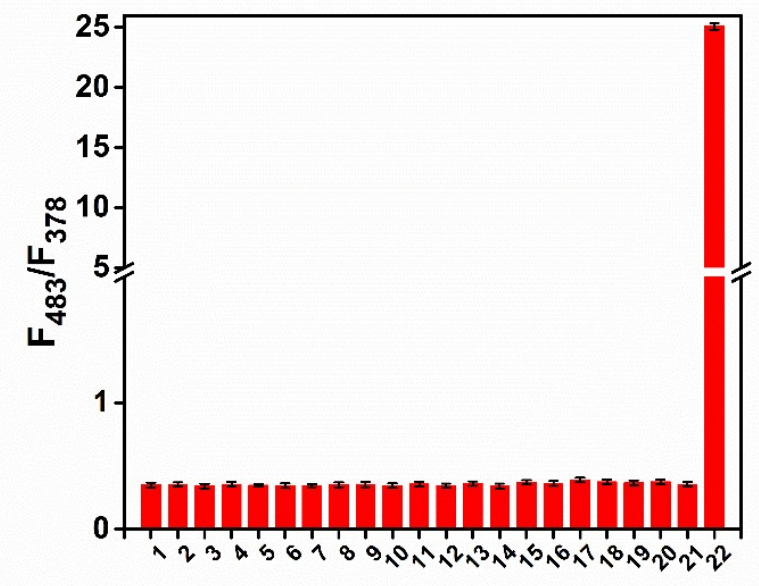

Fig. 2 - Fluorescence intensity ratio changes (based on the peak heights at the maxima, 378 and $483 \mathrm{~nm}$ respectively) with addition of $\mathrm{O}_{2}{ }^{\cdot-}(39$ equiv.) and other interfering reagents

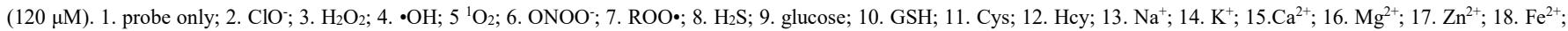
19. $\mathrm{Al}^{3+} ; 20 . \mathrm{Cu}^{2+} ; 21 . \mathrm{Fe}^{3+} ; 22 . \mathrm{O}_{2}{ }^{\circ-} . \lambda_{\text {ex }}=310 \mathrm{~nm}$. Error bar represents s.d.. Slit widths: ex $=8 \mathrm{~nm}, \mathrm{em}=5 \mathrm{~nm} .30$ min wait between measurements.

With this research we have developed an ESIPT-based fluorescence probe (HMBT-LW) for the selective and sensitive detection of $\mathrm{O}_{2}{ }^{-}$. Sadly, the excitation wavelength for HMBT-LW is too short to enable its use in cellular imaging experiments. However, we are currently exploring related ESIPT based systems with longer excitation wavelengths that are more suitable for cellular imaging experiments. In summary HMBT-LW provides a platform on which it will be possible to develop long wavelength ESIPT-based fluorescence probes for the ratiometric selective and sensitive detection of $\mathrm{O}_{2}{ }^{-*}$.

\section{Acknowledgements}

LW wishes to thank China Scholarship Council and the University of Bath for supporting his PhD work in the UK. We would like to thank the EPSRC and the University of Bath for funding. TDJ wishes to thank the Royal Society for a Wolfson Research Merit Award. NMR characterisation facilities were provided through the Chemical Characterisation and Analysis Facility (CCAF) at the University of Bath (www.bath.ac.uk/ccaf). The EPSRC UK National Mass Spectrometry Facility at Swansea University is thanked for analyses. All data supporting this study are provided as supplementary information accompanying this paper (ESI $\dagger$ ).

\section{Notes and references}

1. P. Held, An Introduction to Reactive Oxygen Species - Measurement of ROS in Cells, BioTek Instruments, 2010.

2. J. F. Turrens, J. Physiolo., 2003, 552, 335-344.

3. S. Rose, S. Melnyk, O. Pavliv, S. Bai, T. G. Nick, R. E. Frye and S. J. James, Transl. Psychiatry, 2012,2 , e134.

4. R. A. Cairns, I. S. Harris and T. W. Mak, Nat. Rev. Cancer, 2011, 11, 85-95.

5. C. Gorrini, I. S. Harris and T. W. Mak, Nat. Rev. Drug Discovery, 2013, 12, 931-947.

6. M. H. Lee, J. S. Kim and J. L. Sessler, Chem, Soc. Rev. 2015, 44, 4185-4191.

7. A. C. Sedgwick, L. Wu, H.-H. Han, S. D. Bull, X.-P. He, T. D. James, J. L. Sessler, B. Z. Tang, H. Tian and J. Yoon, Chem. Soc. Rev., 2018, DOI: 10.1039/C8CS00185E.

8. L. Wu, H.-H. Han, L. Liu, J. E. Gardiner, A. C. Sedgwick, C. Huang, S. D. Bull, X.-P. He and T. D. James, Chem. Commun., 2018, 54, 11336-11339.

9. L. Wu, Y. Wang, M. Weber, L. Liu, A. C. Sedgwick, S. D. Bull, C. Huang and T. D. James, Chem. Commun., 2018, 54, 99539956.

10. L. Wu, Q. Yang, L. Liu, A. C. Sedgwick, A. J. Cresswell, S. D. Bull, C. Huang and T. D. James, Chem. Commun., 2018, 54, 8522-8525.

11. A. C. Sedgwick, X. Sun, G. Kim, J. Yoon, S. D. Bull and T. D. James, Chem. Commun., 2016, 52, 12350-12352.

12. P. Li, W. Zhang, K. Li, X. Liu, H. Xiao, W. Zhang and B. Tang, Anal. Chem., 2013, 85, 9877-9881.

13. X. Han, R. Wang, X. Song, F. Yu, C. Lv and L. Chen, Biomaterial, 2018, 156, 134-146.

14. Y. Lv, D. Cheng, D. Su, M. Chen, B.-C. Yin, L. Yuan and X.-B. Zhang, Chem. Sci., 2018, 9, 7606-7613.

15. J. J. Hu, N.-K. Wong, S. Ye, X. Chen, M.-Y. Lu, A. Q. Zhao, Y. Guo, A. C.-H. Ma, A. Y.-H. Leung and J. Shen and D. Yang, J. Am. Chem. Soc., 2015, 137, 6837-6843.

16. D. P. Murale, H. Kim, W. S. Choi and D. G. Churchill, Org. Lett., 2013, 15, 3946-3949.

17. D. Lu, L. Zhou, R. Wang, X.-B. Zhang, L. He, J. Zhang, X. Hu, and W. Tan, Sen. Actuators. B, 2017, 250, $259-266$.

18. A. Sytnik and M. Kasha, Proc. Natl. Acad. Sci. U. S. A., 1994, 91, 8627-8630.

19. X. Yang, Y. Guo and R. M. Strongin, Angew. Chem. Int. Ed., 2011, 50, 10690-10693. 